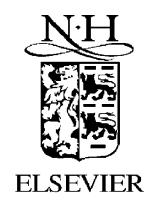

Journal of Geometry and Physics 39 (2001) 276-300

JOURNAL OF

\title{
Conformal Hamiltonian systems
}

\author{
Robert McLachlan*, Matthew Perlmutter \\ IFS, Massey University, Palmerston North, New Zealand 5301
}

Received 4 August 2000; received in revised form 23 January 2001

\begin{abstract}
Vector fields whose flow preserves a symplectic form up to a constant, such as simple mechanical systems with friction, are called "conformal". We develop a reduction theory for symmetric conformal Hamiltonian systems, analogous to symplectic reduction theory. This entire theory extends naturally to Poisson systems: given a symmetric conformal Poisson vector field, we show that it induces two reduced conformal Poisson vector fields, again analogous to the dual pair construction for symplectic manifolds. Conformal Poisson systems form an interesting infinite-dimensional Lie algebra of foliate vector fields. Manifolds supporting such conformal vector fields include cotangent bundles, Lie-Poisson manifolds, and their natural quotients. (๐ 2001 Elsevier Science B.V. All rights reserved.
\end{abstract}

MSC: 53C57; 58705

Sub. Class.: Dynamical systems

Keywords: Hamiltonian systems; Conformal Poisson structure

\section{Introduction}

The fields of symplectic geometry and geometric mechanics are closely linked, indeed almost synonymous $[10,14]$. One of their most important features is that the symplectic diffeomorphisms on a manifold form a group. This property can be taken as the definition of "geometry;" it is natural to try and generalize it. In 1913 Cartan [5,24] found that, subject to certain restrictions, there are just six classes of groups of diffeomorphisms on any manifold, namely (i) Diff itself; (ii) Diff $\omega$, the diffeomorphisms preserving a symplectic 2-form $\omega$; (iii) Diff $_{\mu}$, the diffeomorphisms preserving a volume form $\mu$; (iv) Diff ${ }_{\alpha}$, the diffeomorphisms preserving a contact form $\alpha$ up to a function; and the conformal groups, (v) $\operatorname{Diff}_{\omega}^{c}$ and (vi) $\operatorname{Diff}_{\mu}^{c}$, the diffeomorphisms preserving the form $\omega$ or $\mu$ up to a constant. Classes (i)-(iv)

\footnotetext{
* Corresponding author.

E-mail addresses: r.mclachlan@massey.ac.nz (R. McLachlan), m.perlmutter@massey.ac.nz (M. Perlmutter).
} 
are widely studied in dynamical systems and for their group-theoretic properties [2]. The conformal groups are much less studied yet form natural generalizations of Diff $\mu$ and Diff $\omega$. Two references are [18], in which the quadratic conformal symplectic maps are classified, and [6], in which the symmetry of the Lyapunov spectrum of conformal symplectic systems is studied. It is frequently mentioned that the Lorenz system has constant divergence and that its flow lies in Diff ${ }_{\mu}^{c}$, but this is not usually regarded as being very significant. In this paper we explore some of the geometry and dynamics of the conformal symplectic group $\operatorname{Diff}_{\omega}^{c}$. (Some implications for the numerical solution of such systems were discussed in [15].) ${ }^{1}$

In the simplest case, namely when the manifold is $\mathbb{R}^{2 n}$ with coordinates $(q, p)$ and $\omega=$ $\mathrm{d} q \wedge \mathrm{d} p$, the conformal vector fields - those whose flow is conformal - have the form:

$$
\dot{q}=\frac{\partial H}{\partial p}, \quad \dot{p}=-\frac{\partial H}{\partial q}+c p,
$$

where $H: \mathbb{R}^{2 n} \rightarrow \mathbb{R}$ is the Hamiltonian. Their flow has the property $\varphi^{*} \omega=\mathrm{e}^{c t} \omega$, so the symplectic inner product of any two tangent vectors contracts exponentially if $c<0$. This case includes simple mechanical systems with friction, such as the Duffing oscillator. If $H=T+V(q), T=\frac{1}{2} p^{t} M(q) p$, then $\dot{H}=c T \leq 0$ when $c<0$ and $M$ is positive definite. Such systems dissipate energy and symplectic area. (In general, however, $\dot{H}$ is not sign definite.) Only this very special type of friction is compatible with the symplectic structure in the required way. In Section 2 we study conformal systems and their algebraic structure on more general symplectic manifolds.

The term 'dissipation' can also refer to a decrease of energy [16], such as in systems with Rayleigh dissipation, $\dot{q}=H_{p}, \dot{p}=-H_{q}-R(q) H_{p}$, for which $\dot{H}=-R(q)\left(H_{p}, H_{p}\right) \leq 0$. (Here $R(q)$ defines a positive metric.) Simple mechanical systems with Rayleigh dissipation are conformal when $R(q)=c M(q)^{-1}$. In general, however, Rayleigh dissipation does not imply any special behavior of the symplectic form.

How is conformal dynamics different from any other kind? This really deserves a major study of its own. One known property is that its eigenvalues at any point, and hence its Lyapunov exponents, sum in pairs to $c$ [6]. This controls the phase portraits near fixed points and bounds the Lyapunov dimension of any attractors to $\leq n$. However, the Lyapunov spectrum is not invariant under homeomorphisms and one would expect that Diff ${ }_{\omega}^{c}$, having Diff $\omega_{\omega}$ as a codimension-1 subgroup, would have similarly constrained dynamics. Motivated by these questions, we consider the geometric structure of conformal systems with symmetry, and how it differs from arbitrary symmetric systems on one hand, and from symmetric Hamiltonian and Poisson systems on the other.

A Hamiltonian system with an appropriate symmetry has the momentum equation $\dot{J}=0$. In the conformal case, we derive in Proposition 7 the modified equation $\dot{J}=c J$. That is, the foliation defined by the level sets of the momentum map is still invariant, although each leaf is no longer fixed.

\footnotetext{
${ }^{1}$ Note that the term 'conformal symplectic' is also used in the literature [10,23] to refer to 2-forms which are not closed but for which there is a function $f$ such that $\mathrm{d}(f \omega)=0$. This type of conformal symplectic dynamics is a re-parameterization in time of an ordinary symplectic flow.
} 
In Section 3 we begin a more detailed reduction and reconstruction theory by studying the case of a $G$-invariant system on $T^{*} G$ : many elements of the standard theory survive in a modified form. Specifically, we find reduced vector fields on each leg of the associated dual pair; left reduction leads to a "conformal Lie-Poisson system," and right reduction leads to the conformal momentum equation $\mathbf{J}=c \mathbf{J}$. (See also [3], in which a special Rayleigh dissipation function also leads to a reduced dissipative system on the dual of the Lie algebra of $G$; these reduced systems are not conformal but do actually preserve momentum, $\dot{\mathbf{J}}=0$.)

Today, the Cartan classification is studied in terms of Lie pseudogroups, one of several possible infinite-dimensional analogs of Lie groups [9,21]. They are said to be Lie because they are defined as the solutions of PDEs, and pseudo because such solutions are in general only local diffeomorphisms, so that composition is only defined when the domains and ranges overlap (thus they are not true groups). A simple example is the (finite-dimensional) Lie pseudogroup on $\mathbb{R}$ defined by $\varphi^{\prime} \varphi^{\prime \prime \prime}-\frac{3}{2} \varphi^{\prime \prime 2}=0$ with solution the local diffeomorphisms $\varphi: x \mapsto(a x+b) /(c x+d)$. The restrictions to the Cartan classification mentioned above are that it applies only to transitive (for all $x, y \in M$ there exists $\varphi \in G$ such that $\varphi(x)=$ $y$ ), primitive (there is no foliation of $M$ which is left invariant by every element of $G$ ), infinite-dimensional Lie pseudogroups $G$ on complex manifolds $M$. In the 1960s gaps were discovered in Cartan's proof, which were corrected by Guillemin et al. [7,20]; the classification was extended to the real case by Shnider [19]. There seems to have been little progress in classifying the general case since then.

The main pseudogroups form the different "kinds of dynamics" that people study complex, volume preserving, symmetric, and so on — and despite the lack of a complete classification, there is nothing to stop us looking for interesting non-primitive pseudogroups as they arise in dynamics. A non-primitive pseudogroup leaves some foliation invariant. For example, in the case of a trivial foliation, $M=L \times F$ where $L$ is a leaf and $F$ is the space of leaves, the maps preserving the foliation have the form $\varphi(x, y)=(f(x, y), g(y))$ and are known as "skew product" systems, introduced by Anzai [1], and studied today in ergodic theory [4] and complex dynamics [8].

In our context, one immediate example of a non-primitive pseudogroup is a Poisson manifold together with its automorphisms, the Poisson maps, which we study in Section 4. The manifold is foliated into symplectic leaves and this foliation is preserved by the Poisson maps. In the general case, the dimension of the leaves may vary from point to point and we have a generalized foliation [10]. (In the case usually studied, each leaf is itself preserved, as in the flows of Hamiltonian ODEs, but this is not necessary.) Poisson manifolds also carry a natural conformal Lie pseudogroup, the maps that preserve the Poisson bracket up to a function of the leaf (i.e., up to a Casimir). It has many subgroups, the most important being that in which the function of the leaf is constant, which we call special conformal. In this section we characterize conformal Poisson maps as leaf-to-leaf conformal symplectic maps; we show that the Hamiltonian and the Poisson vector fields form ideals in the Lie algebra of conformal Poisson vector fields; and we show that when the Poisson bracket is homogeneous of degree not equal to 2 (e.g., in the Lie-Poisson case), the linear radial vector field $x_{i}\left(\partial / \partial x_{i}\right)$ is conformal. 
In Section 5 we study conformal Poisson systems with symmetry. We prove that just as Hamiltonian systems with symmetry on symplectic manifolds reduce to Hamiltonian systems on Poisson manifolds, conformal systems on symplectic manifolds reduce to special conformal systems on Poisson manifolds. We also present a theory of reduction entirely in the Poisson category.

In a sense, the conformal Poisson pseudogroup is one of the simplest non-primitive pseudogroups, in that the foliation has no transverse structure and the induced maps on the leaf spaces are locally unconstrained. We describe the pseudogroup to some extent but several questions remain open, especially that of which Poisson manifolds support a special conformal vector field. Our examples of those that do include exact symplectic manifolds, constant and Lie-Poisson manifolds, Lie algebroids, and a quadratic bracket on $\mathbb{R}^{3}$; reduction by stages gives a more complicated example. In simple cases the vector field which is radial and linear on suitable fibers (known as the Liouville vector field [10], $\dot{q}=0$, $\dot{p}=p$ in the canonical case) is conformal.

\section{Conformal vector fields on exact symplectic manifolds}

Let $M$ be a symplectic manifold with symplectic form $\omega$. If $M$ is exact we write $\omega=-\mathbf{d} \theta$. For a function $H$ on $M$ we denote its Hamiltonian vector field by $X_{H}$. We work in the smooth $\left(C^{\infty}\right)$ category throughout.

Definition 1. The vector field $X^{c}$ is said to be conformal with parameter $c \in \mathbb{R}$ if

$$
\mathbf{L}_{X^{c}} \omega=c \omega .
$$

The diffeomorphism $\varphi^{c}$ is conformal if

$$
\left(\varphi^{c}\right)^{*} \omega=c \omega .
$$

The diffeomorphisms $\varphi^{c}$ form the pseudogroup Diff ${ }_{\omega}^{c}$, one of Cartan's six fundamental classes of primitive pseudogroups.

\section{Proposition 1.}

1. The time-t flow of $X^{c}$ is conformal with parameter $\mathrm{e}^{c t}$.

2. $(M, \omega)$ admits a conformal vector field with parameter $c \neq 0$ if and only if $M$ is exact.

3. Given $H \in C^{\infty}(M)$ and $M$ exact, the vector field $X_{H}^{c}$ defined by

$$
\mathbf{i}_{X_{H}^{c}} \omega=\mathbf{d} H-c \theta
$$

is conformal.

4. If, in addition, $H^{1}(M)=0$, then given $X^{c}$ there exists a function $H$ such that $X^{c}=X_{H}^{c}$, and the set of conformal vector fields on $M$ is given by

$$
\left\{X_{H}+c Z: H \in C^{\infty}(M)\right\},
$$


where $Z$ is defined by

$$
\mathbf{i}_{Z} \omega=-\theta \text {. }
$$

\section{Proof.}

1. Let $\varphi_{t}$ denote the flow of $X^{c}$. By the Lie derivative theorem, we have $(\mathrm{d} / \mathrm{d} t) \varphi_{t}^{*} \omega=$ $\varphi_{t}^{*} \mathbf{L}_{X^{c}} \omega=c \varphi_{t}^{*} \omega$ which has the unique solution $\varphi_{t}^{*} \omega=\mathrm{e}^{c t} \omega$.

2. Using the homotopy formula for Lie derivatives, we see that $X^{c}$ is conformal on $(M, \omega)$ if and only if $\mathbf{d i}_{X^{c}} \omega=c \omega$. Therefore, if $c \neq 0$, we see that $\omega$ must be exact so that $\omega=-\mathbf{d} \theta$ for some 1-form $\theta$. Eq. (2.1) then reads

$$
\mathbf{d i}_{X^{c}} \omega=-c \mathbf{d} \theta .
$$

3. $\mathbf{L}_{X_{H}^{c}} \omega=\mathbf{d i}_{X_{H}^{c}} \omega=-c \mathbf{d} \theta=c \omega$, as required.

4. Notice that $\mathbf{L}_{Z} \omega=\mathbf{d i}_{Z} \omega=-\mathbf{d} \theta=\omega$. Furthermore, for any $H \in C^{\infty}(M)$, we have $\mathbf{L}_{X_{H}} \omega=0$ and so clearly $Z+X_{H}$ is conformal with parameter 1 . Conversely, given $X^{c}$, if $H^{1}(M)=0$, from Eq. (2.6) there exists $H$ such that $\mathbf{i}_{X^{c}} \omega=\mathbf{d} H-c \theta$, from which we conclude, by non-degeneracy of $\omega$, that $X^{c}=X_{H}+c Z$.

Following [10] we shall call $Z$ the Liouville vector field.

Proposition 2. The conformal vector fields on $(M,-\mathbf{d} \theta)$ form a Lie algebra of vector fields with the Hamiltonian vector fields as an ideal. If $H^{1}(M)=0$, the quotient of the conformal vector fields by the Hamiltonian vector fields is a one-dimensional subspace parameterized by the constants $c$.

\section{Proof.}

$$
\mathbf{L}_{X^{c}} \mathbf{i}_{X_{h}} \omega=\mathbf{i}_{\left[X^{c}, X_{h}\right]} \omega+\mathbf{i}_{X_{h}} \mathbf{L}_{X^{c}} \omega=\mathbf{i}_{\left[X^{c}, X_{h}\right]} \omega+c \mathbf{i}_{X_{h}} \omega=\mathbf{i}_{\left[X^{c}, X_{h}\right]} \omega+c \mathbf{d} h
$$

from which we conclude

$$
\mathbf{i}_{\left[X^{c}, X_{h}\right]} \omega=\mathbf{L}_{X^{c}} \mathbf{d} h-c \mathbf{d} h=\mathbf{d}\left(X^{c}(h)-c h\right) .
$$

Thus, $\left[X_{h}, X^{c}\right]=X_{-\left(X^{c}(h)-c h\right)}$. Finally, if $H^{1}(M)=0$, we know that every conformal vector field can be written as $X_{H}+c Z$ for some Hamiltonian and a unique $c \in \mathbb{R}$, from which the last statement follows.

Next, we consider the effect of the flow of $X^{c}$ on the canonical Poisson bracket.

Proposition 3. The vector field $X^{c}$ is conformal if and only if for all $f, g \in C^{\infty}(M)$,

$$
X^{c}\{f, g\}=\left\{X^{c} f, g\right\}+\left\{f, X^{c} g\right\}-c\{f, g\} .
$$

The diffeomorphism $\varphi^{c}$ is conformal if and only if

$$
\left\{f \circ \varphi^{c}, g \circ \varphi^{c}\right\}=c\{f, g\} \circ \varphi^{c} .
$$


Proof. We compute

$$
\begin{aligned}
X^{c}\{f, g\} & =\mathbf{L}_{X^{c}}\left(\omega\left(X_{f}, X_{g}\right)\right)=\mathbf{L}_{X^{c}}\left(\mathbf{i}_{X_{g}}\left(\mathbf{i}_{X_{f}} \omega\right)\right) \\
& =\mathbf{i}_{\mathbf{L}_{X^{c}}\left(X_{g}\right)}\left(\mathbf{i}_{X_{f}} \omega\right)+\mathbf{i}_{X_{g}}\left(\mathbf{i}_{\mathbf{L}_{X^{c}}\left(X_{f}\right)} \omega\right)+\mathbf{i}_{X_{g}}\left(\mathbf{i}_{X_{f}}\left(\mathbf{L}_{X^{c}} \omega\right)\right) \\
& =\mathbf{i}_{\left[X^{c}, X_{g}\right]}\left(\mathbf{i}_{X_{f}} \omega\right)+\mathbf{i}_{X_{g}}\left(\mathbf{i}_{\left[X^{c}, X_{f}\right]} \omega\right)+c \omega\left(X_{f}, X_{g}\right) .
\end{aligned}
$$

Using $\left[X^{c}, X_{g}\right]=X_{X^{c}}(g)-c g$ from the previous proposition, we can rewrite the first term as $\omega\left(X_{f}, X_{X^{c}(g)}\right)-c\{f, g\}$ and similarly for the second term from which the proposition is now immediate.

In the case that $M$ is a canonical cotangent bundle, the conformal vector field $Z$ has a particularly simple form. The following proposition shows how simple mechanical systems with linear friction, Eq. (1.1), are conformal.

Proposition 4. Let $(M, \omega)=\left(T^{*} Q,-\mathbf{d} \Theta\right)$. Then $Z$ is tangent to the fibers of the cotangent bundle and is linear and radial on the fibers; in local coordinates $(q, p)$,

$$
Z=p \frac{\partial}{\partial p}
$$

Proof. On a linear space, the linear radial vector field is characterized as the unique vector field which leaves all linear functions invariant. Any function linear on the fibers of $T^{*} Q$ can be represented as

$$
P_{X}\left(\alpha_{q}\right)=\left\langle\alpha_{q}, X(q)\right\rangle
$$

for some vector field $X$ on $Q$. It suffices to check that $Z\left(P_{X}\right)=P_{X}$. We have

$$
Z\left(P_{X}\right)=\mathbf{d} P_{X} \cdot Z=\omega\left(X_{P_{X}}, Z\right)=\theta\left(X_{P_{X}}\right)=P_{X},
$$

where $X_{P_{X}}$ is the Hamiltonian vector field corresponding to the function $P_{X}$. The third equality follows from the definition of $Z$ and the final equality holds since the flow of $X_{P_{X}}$ is the cotangent lift of the flow of $X$ on $Q$. The expression $Z=p(\partial / \partial p)$ now follows directly or can be found by solving $\mathbf{i}_{Z}(\mathrm{~d} q \wedge \mathrm{d} p)=p \mathrm{~d} q$.

Proposition 5. Consider the time-dependent diffeomorphism $\psi_{t}: T^{*} Q \rightarrow T^{*} Q$ given by

$$
\psi_{t}\left(\alpha_{q}\right)=\mathrm{e}^{-c t} \alpha_{q} .
$$

Then, $X_{H}^{c}$ is the pull back by $\psi_{t}$ of a time-dependent Hamiltonian vector field on $T^{*} Q$. In local coordinates $(q, p)$ on $T^{*} Q$ the Hamiltonian is given by

$$
\tilde{H}(\tilde{q}, \tilde{p})=\mathrm{e}^{-c t} H\left(\tilde{q}, \mathrm{e}^{c t} \tilde{p}\right),
$$

where $(\tilde{q}, \tilde{p})=\psi_{t}(q, p)=\left(q, \mathrm{e}^{-c t} p\right)$.

Proof. It suffices to work in local coordinates $(q, p)$. Hamilton's equations in $(\tilde{q}, \tilde{p})$ for $\tilde{H}$ are given by

$$
\dot{\tilde{q}}=\frac{\partial \tilde{H}}{\partial \tilde{p}}=\frac{\partial H}{\partial p}=\dot{q}, \quad \dot{\tilde{p}}=-\frac{\partial \tilde{H}}{\partial \tilde{q}}=\mathrm{e}^{-c t} \frac{\partial H}{\partial q} .
$$


Now, since $\tilde{p}=\mathrm{e}^{-c t} p$ we also have

$$
\dot{\tilde{p}}=\mathrm{e}^{-c t}(\dot{p}-c p)=\mathrm{e}^{-c t}\left(-\frac{\partial H}{\partial q}\right)
$$

from which the claim follows.

We now consider an action of a Lie group $G$ on the exact symplectic manifold $(M,-\mathbf{d} \theta)$ leaving the 1-form $\theta$ invariant. It is well known that such an action admits an $\mathrm{Ad}^{*}$-equivariant momentum map. Suppose this action also leaves invariant the function $H \in C^{\infty}(M)$. In the case of Hamiltonian vector fields, we know that $X_{H}$ is tangent to the level surfaces of the momentum map, $\mathbf{J}^{-1}(\mu)$. This is not the case for conformal vector fields associated to the $G$-invariant function $H$. However, there is a simple momentum equation satisfied by such systems which constrains the motion to a ray of momentum values defined by the initial momentum.

Proposition 6. For any $c$, and any $G$-invariant function $H \in C^{\infty}(M), X_{H}^{c}$ is a $G$-invariant vector field.

\section{Proof.}

$$
\varphi_{g}^{*}\left(\mathbf{i}_{X_{H}^{c}} \omega\right)=\mathbf{i}_{\varphi_{g}^{*}\left(X_{H}^{c}\right)}\left(\varphi_{g}^{*} \omega\right)=\mathbf{i}_{\varphi_{g}^{*}\left(X_{H}^{c}\right)} \omega .
$$

On the other hand,

$$
\varphi_{g}^{*}\left(\mathbf{i}_{X_{H}^{c}} \omega\right)=\varphi_{g}^{*}(\mathbf{d} H-c \theta)=\mathbf{d} H-c \theta=\mathbf{i}_{X_{H}^{c}} \omega
$$

so that $\mathbf{i}_{\varphi_{g}^{*} X_{H}^{c}} \omega=\mathbf{i}_{X_{H}^{c}} \omega$, from which, by non-degeneracy of $\omega$, the proposition follows.

Remark. Notice that on a cotangent bundle the conformal vector field $Z$ (Eq. (2.9)) is invariant with respect to any cotangent lifted diffeomorphism.

Proposition 7 (The conformal momentum equation). Let $G$ be a Lie group acting on the left of $M, G \times M \rightarrow M$, leaving the 1-form $\theta$ invariant. Let $H$ be a $G$-invariant function on M. We know that $X_{H}^{c}$ is a G-invariant vector field. Moreover, we have for all $\xi \in \mathfrak{g}$,

$$
\mathbf{d} J(\xi) \cdot X_{H}^{c}=c J(\xi)
$$

and so

$$
T \mathbf{J} \cdot X_{H}^{c}=c \mathbf{J}
$$

where, as usual, $J(\xi)=\langle\mathbf{J}, \xi\rangle$.

Proof. Since $G$ leaves $\theta$ invariant, we have, for each $\xi \in \mathfrak{g}$,

$$
\mathbf{L}_{\xi_{M}} \theta=0=\mathbf{i}_{\xi_{M}} \mathbf{d} \theta+\mathbf{d i}_{\xi_{M}} \theta
$$

where $\xi_{M}$ is the infinitesimal generator for the action of $G$ on $M$. This implies $J(\xi)=\mathbf{i}_{\xi_{M}} \theta$. 
Now, since $X_{H}^{c}=X_{H}+c Z$, we have

$$
\mathbf{d} J(\xi) \cdot\left(X_{H}+c Z\right)=-\mathbf{d} H \cdot \xi_{M}+\omega\left(\xi_{M}, c Z\right)=-c \mathbf{i}_{Z} \omega\left(\xi_{M}\right)=c \theta\left(\xi_{M}\right)=c J(\xi),
$$

as required.

Example 1 (Angular momentum). Consider $M=T^{*}\left(\mathbb{R}^{3}\right)$ with canonical symplectic form $\mathrm{d} q \wedge \mathrm{d} p$. Since $H^{1}\left(T^{*}\left(\mathbb{R}^{3}\right)\right)=0$, any conformal vector field is of the form $X_{H}^{c}=X_{H}+c Z$ for some function $H$. Suppose $H$ is $\mathrm{SO}(3)$-invariant for the cotangent lifted action of $\mathrm{SO}(3)$ on $T^{*} \mathbb{R}^{3}$, i.e. $H=h\left(\frac{1}{2}|q|^{2}, \frac{1}{2}|p|^{2}, q \cdot p\right)$. The momentum map for this action is given by $\mathbf{J}(q, p)=q \times p$, the classical angular momentum. Then the equations of motion are

$$
\dot{q}=h_{2} p+h_{3} q, \quad \dot{p}=-h_{1} q-h_{3} p+c p
$$

so

$$
\frac{\mathrm{d}}{\mathrm{d} t} \mathbf{J}=\frac{\mathrm{d}}{\mathrm{d} t}(q \times p)=q \times \dot{p}+\dot{q} \times p=c q \times p=c \mathbf{J},
$$

as in Proposition 7.

In contrast to the Hamiltonian case, $X_{H}$ is not in general a symmetry of $X_{H}^{c}$ and so there is no 'momentum' equation for energy. Instead we have $\dot{H}=Z(H) \neq 0$ which can have, in general, any sign.

In the case of a free group action, the momentum equation $\mathbf{J}=c \mathbf{J}$ produces $\operatorname{dim} G$ eigenvalues (and hence Lyapunov exponents) equal to $c$. Since eigenvalues sum in pairs to $c$, there are also $\operatorname{dim} G$ eigenvalues equal to 0 , just as in the symplectic case. An example of this in the case of linear momentum can be seen in the computations in [6].

By analogy with the Hamiltonian case, we consider the dual pair [22]

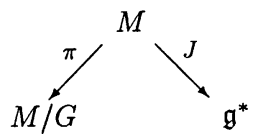

and ask what can be said about reduction and reconstruction of the symmetric conformal vector field $X_{H}^{c}$. The momentum equation (2.10) implies that $X_{H}^{c}$ projects to a well-defined vector field on $\mathfrak{g}^{*}$ under the derivative of the momentum map since $T \mathbf{J} \cdot X_{H}^{c}$ is constant along the fibers of $\mathbf{J}$. Furthermore, we assume the momentum map is both surjective onto $\mathfrak{g}^{*}$ and submersive. Note that this is always the case for cotangent lifted actions. Thus, $T \mathbf{J} \cdot X_{H}^{c}$ is a vector field on $\mathfrak{g}^{*}$. (In the Hamiltonian case, of course, this dropped vector field is zero.) The left-hand side of the dual pair also inherits a vector field, since $X_{H}^{c}$ is $G$-invariant. We will see in Section 4 that this vector field, $T \pi \cdot X_{H}^{c}$, is also conformal relative to the reduced Poisson structure on $M / G$, and that it is generated by the dropped function $h \in C^{\infty}(M / G)$ defined by $H=h \circ \pi$. However, we first study in detail the "Lie-Poisson" case, $M=T^{*} G$ with canonical symplectic form $\omega=-\mathbf{d} \Theta$. 


\section{Conformal Lie-Poisson reduction and reconstruction}

We first establish some notation that will be used throughout this section. For any $g \in G$, $L_{g}$ and $R_{g}$ will denote left and right translation on the group, respectively. We will use concatenated notation for the corresponding left and right tangent and cotangent lifted actions, $G \times T G \rightarrow T G, T G \times G \rightarrow T G, G \times T^{*} G \rightarrow T^{*} G$, and $T^{*} G \times G \rightarrow T^{*} G$, respectively. Thus, for $v_{h} \in T_{h} G, \alpha_{h} \in T_{h}^{*} G$, and $g \in G$ we have

$$
\begin{aligned}
g \cdot v_{h} & :=T_{h} L_{g} \cdot v_{h}, \\
v_{h} \cdot g & :=T_{h} R_{g} \cdot v_{h}, \\
g \cdot \alpha_{h} & :=T_{g h}^{*} L_{g^{-1}} \alpha_{h} \in T_{g h}^{*} G, \\
\alpha_{h} \cdot g & :=T_{g h}^{*} R_{g^{-1}} \alpha_{h} \in T_{h g}^{*} G .
\end{aligned}
$$

We also let $\tau: T^{*} G \rightarrow G$ be the cotangent bundle projection, $\pi: T^{*} G \rightarrow \mathfrak{g}^{*}$ be the quotient for the left cotangent lifted action, and $\pi_{\mathrm{R}}: T^{*} G \rightarrow \mathfrak{g}^{*}$ be the quotient for the right cotangent lifted action. Thus $\pi\left(\alpha_{h}\right)=h^{-1} \cdot \alpha_{h}$ and similarly for $\pi_{R}$.

We know that for a left $G$-invariant function $H$ on $T^{*} G$, the conformal vector field $X_{H}^{c}$ is left $G$-invariant and therefore drops to a vector field on

$$
T^{*} g / g \simeq \mathfrak{g}^{*} .
$$

It turns out that the dropped vector field is also conformal relative to the Lie-Poisson structure on $\mathfrak{g}^{*}$ and is related to the standard Lie-Poisson Hamiltonian vector field in a simple way. Recall that the Lie-Poisson bracket for the left quotient is given by

$$
\{f, g\}(\mu)=-\left\langle\mu,\left[\frac{\delta f}{\delta \mu}, \frac{\delta g}{\delta \mu}\right]\right\rangle,
$$

where $(\delta f / \delta \mu)$ is the unique element of $\mathfrak{g}$ such that

$$
\left\langle v, \frac{\delta f}{\delta \mu}\right\rangle=\mathbf{d} f(\mu) \cdot v
$$

holds for all $v \in \mathfrak{g}^{*}$. The Hamiltonian vector field associated to the function $h$ is then $X_{h}^{-}(\mu)=\operatorname{ad}_{\delta h / \delta \mu}^{*} \mu$.

Theorem 1 (Conformal Lie-Poisson reduction). We have

$$
T \pi \cdot X_{H}^{c}=X_{h}^{c} \circ \pi
$$

where $X_{h}^{c}$ is the vector field on $\mathfrak{g}^{*}$ given by

$$
X_{h}^{c}(\mu)=X_{h}^{-}(\mu)+c \mu .
$$

Proof. Let $H$ and $F$ be two $G$-invariant functions on $T^{*} G$. We let $H=h \circ \pi$ and $F=f \circ \pi$ define the functions $h$ and $f$ on $\mathfrak{g}^{*}$. First notice that the function $\mathbf{d} F \cdot X_{H}^{c}$ is $G$-invariant as 
well since $\mathbf{d} F$ is $G$-invariant, and from Proposition 6, we know that $X_{H}^{c}$ is $G$-invariant. Fix $\alpha_{g} \in T^{*} G$ and let $\mu=\pi\left(\alpha_{g}\right)$. We then have,

$$
\mathbf{d} F\left(\alpha_{g}\right) \cdot X_{H}^{c}\left(\alpha_{g}\right)=\mathbf{d} f(\mu) \cdot T \pi \cdot X_{H}^{c}\left(\alpha_{g}\right),
$$

so that we can read off the dropped vector field by computing the left-hand side. From the definition of $X_{H}^{c}$ we have,

$$
\mathbf{d} F \cdot X_{H}^{c}=\mathbf{d} F \cdot\left(X_{H}+c Z\right)=\{F, H\}-c \mathbf{i}_{Z} \omega\left(X_{F}\right)=\{F, H\}+c \Theta\left(X_{F}\right) .
$$

We can immediately express the first term in terms of the quotient functions using the Lie-Poisson reduction theorem. We turn attention to the second term. Recall that $X_{F}$ is a $G$-invariant vector field on $T^{*} G$ and is tangent to the level surfaces of the momentum map. To compute

$$
\Theta\left(X_{F}\right)\left(\alpha_{g}\right)=\left\langle\alpha_{g}, T \tau \cdot\left(X_{F}\left(\alpha_{g}\right)\right)\right\rangle,
$$

we use the following facts from the reconstruction theory for Lie-Poisson dynamics. The integral curve of $X_{F}$ through $\alpha_{g}$ is constructed by first computing the reduced trajectory

$$
\dot{\mu}=X_{f}(\mu), \quad \mu(0)=\mu,
$$

which determines the curve in $\mathfrak{g}$ given by $\xi(t)=\left.(\delta f / \delta \mu)\right|_{\mu=\mu(t)}$ and then solving the time-dependent differential equation on $G$ given by

$$
\dot{g}(t)=g(t) \cdot \xi(t), \quad g(0)=g .
$$

The integral curve through $\alpha_{g}$ is then given by $g(t) \cdot \mu(t)$. We then have

$$
T_{\alpha_{g}} \tau\left(X_{F}\left(\alpha_{g}\right)\right)=\left.\frac{\mathrm{d}}{\mathrm{d} t}\right|_{t=0} \tau(g(t) \cdot \mu(t))=\left.\frac{\mathrm{d}}{\mathrm{d} t}\right|_{t=0} g(t)=g \cdot \frac{\delta f}{\delta \mu},
$$

so that, from Eq. (3.8), we have

$$
\Theta\left(X_{F}\right)\left(\alpha_{g}\right)=\left\langle\alpha_{g}, g \cdot \frac{\delta f}{\delta \mu}\right\rangle=\left\langle g^{-1} \cdot \alpha_{g}, \frac{\delta f}{\delta \mu}\right\rangle=\left\langle\mu, \frac{\delta f}{\delta \mu}\right\rangle .
$$

Following Eqs. (3.6) and (3.7) we have

$$
\mathbf{d} f(\mu) \cdot T_{\alpha_{g}} \pi \cdot X_{H}^{c}\left(\alpha_{g}\right)=\{F, H\}\left(\alpha_{g}\right)+c \Theta\left(X_{F}\right)=\mathbf{d} f(\mu) \cdot X_{h}^{-}(\mu)+\mathbf{d} f(\mu) \cdot c \mu .
$$

Since $f \in C^{\infty}\left(\mathfrak{g}^{*}\right)$ is arbitrary, we conclude, again from Eq. (3.6), and from the definition of $X_{h}^{c}$,

$$
T_{\alpha_{g}} \pi \cdot X_{H}^{c}\left(\alpha_{g}\right)=X_{h}^{c} \circ \pi\left(\alpha_{g}\right)=X_{h}^{-}(\mu)+c \mu .
$$

The reduced vector field is conformal in the following sense.

Proposition 8. Let $\{$,$\} be the left Lie-Poisson bracket on \mathfrak{g}^{*}$. For all $f, g \in C^{\infty}\left(\mathfrak{g}^{*}\right)$, the vector field $X_{h}^{c}$ obtained through reduction satisfies

$$
X_{h}^{c}\{f, g\}=\left\{X_{h}^{c} f, g\right\}+\left\{f, X_{h}^{c} g\right\}-c\{f, g\} .
$$


Proof. Let $R^{c}(\mu)=c \mu$ so that $X_{h}^{c}(\mu)=X_{h}^{-}(\mu)+R^{c}(\mu)$. Since $X_{h}^{-}$is Hamiltonian, it suffices to check that

$$
R^{c}\{f, g\}(\mu)=\left\{R^{c} f, g\right\}+\left\{f, R^{c} g\right\}-c\{f, g\} .
$$

Now since the phase space $\mathfrak{g}^{*}$ is linear, it suffices to check Eq. (3.10) on linear functions. For a linear function $f$ note that we have $f(\mu)=\mathbf{d} f(\mu) \cdot \mu$ so that

$$
R^{c}(f)(\mu)=\mathbf{d} f(\mu) \cdot c \mu=c f(\mu) .
$$

Furthermore the Lie-Poisson bracket of two linear functions $f$ and $g$ is again a linear function corresponding to the bracket of the Lie algebra elements corresponding to $f$ and $g$. Thus $R^{c}\{f, g\}=c\{f, g\}$ and therefore the left-hand side of Eq. (3.10) is $c\{f, g\}$ while the right-hand side is

$$
\left\{R^{c} f, g\right\}+\left\{f, R^{c} g\right\}-c\{f, g\}=\{c f, g\}+\{f, c g\}-c\{f, g\}=c\{f, g\},
$$

as required.

We now reconstruct the solution to the conformal Hamiltonian system on $T^{*} G$ from the reduced vector fields on the legs of the dual pair.

Theorem 2. Let $H=h \circ \pi$ be a left-invariant function on $T^{*} G$ and denote by $X_{H}^{c}$ the corresponding conformal vector field. Fix a point $\alpha_{g_{0}} \in T_{g_{0}}^{*} G$ and denote by $\alpha_{g}(t)$ the integral curve of $X_{H}^{c}$ through $\alpha_{g_{0}}$. Denote by $\nu_{0} \in \mathfrak{g}^{*}$ the right translation of $\alpha_{g_{0}}$ to the identity, i.e. $v_{0}=\alpha_{g_{0}} \cdot g_{0}^{-1}$. Let $\mu(t)$ be the solution to the conformal Lie-Poisson equation $\dot{\mu}=X_{h}^{c}(\mu), \mu(0)=\pi\left(\alpha_{g_{0}}\right)$. Then the reconstructed integral curve is given by

$$
\left(\mathrm{e}^{c t} v_{0}\right) \cdot g(t)
$$

where $g(t)$ is a curve through $g$ satisfying the differential equation on G given by

$$
g(t)^{-1} \cdot \dot{g}(t)=\left.\frac{\delta h}{\delta \mu}\right|_{\mu=\mu(t)} .
$$

Proof. From the momentum equation (2.10) for conformal vector fields we know that

$$
\mathbf{J}\left(\alpha_{g}(t)\right)=\pi_{R}\left(\alpha_{g}(t)\right)=\alpha_{g}(t) \cdot g(t)^{-1}=\mathrm{e}^{c t} \nu_{0} .
$$

Therefore $\alpha_{g}(t)=\mathrm{e}^{c t} \nu_{0} \cdot g(t)$. On the other hand, we know from Theorem 1 that the integral curve for $X_{H}^{c}$ projects onto the integral curve for $X_{h}^{c}$ under the map $\pi: T^{*} G \rightarrow \mathfrak{g}^{*}$. We can use this fact to derive a differential equation satisfied by $g(t)$. We have

$$
\mu(t)=\pi\left(\alpha_{g}(t)\right)=g(t)^{-1} \cdot\left(\mathrm{e}^{c t} \nu_{0} \cdot g(t)\right)=\operatorname{Ad}_{g(t)}^{*} v(t),
$$

where $v(t)=\mathrm{e}^{c t} v_{0}$. Equivalently,

$$
v(t)=\operatorname{Ad}_{g(t)^{-1}}^{*} \mu(t)
$$


Taking the time derivative of both sides of this equation, and evaluating at time $t$, we obtain,

$$
c v(t)=\operatorname{Ad}_{g(t)^{-1}}^{*}\left(\operatorname{ad}_{-\xi(t)}^{*} \mu(t)+\dot{\mu}(t)\right)=\operatorname{Ad}_{g(t)^{-1}}^{*}\left(\operatorname{ad}_{-\xi(t)}^{*} \mu(t)+X_{h}^{-}(\mu(t))+c \mu(t)\right),
$$

where $\xi(t):=g(t)^{-1} \cdot \dot{g}(t)$. The left-hand side is in fact $c \operatorname{Ad}_{g(t)^{-1}}^{*} \mu(t)$ so we get

$$
\operatorname{Ad}_{g(t)^{-1}}^{*}\left(\operatorname{ad}_{-\xi(t)}^{*} \mu(t)+X_{h}^{-}(\mu(t))\right)=0
$$

which is equivalent to

$$
\operatorname{ad}_{-\xi(t)}^{*} \mu(t)+\operatorname{ad}_{\left.(\delta h / \delta \mu)\right|_{\mu=\mu(t)} ^{*}}^{*} \mu(t)=0 .
$$

Therefore $\xi(t)=\left.(\delta h / \delta \mu)\right|_{\mu=\mu(t)}$, from which the statement of the theorem now follows.

Remark. Notice that $\mu(t)$ satisfies the conformal Lie-Poisson equations and as a result the $g(t)$ dynamics is affected by the conformal constant $c$. When $c=0$ the $\mu(t)$ dynamics reduces to Hamiltonian Lie-Poisson dynamics and the above algorithm recovers the known reconstruction equations in that case.

\section{Conformal vector fields on Poisson manifolds}

The reduced vector field $X_{h}^{c}$ of the previous section has the property that its flow preserves the Lie-Poisson bracket on $\mathfrak{g}^{*}$ up to a constant. Conformal vector fields on symplectic manifolds also preserve the (canonical) Poisson bracket up to a constant. Therefore, we expect that the results of the previous section can be generalized from symplectic to Poisson manifolds. One substantially new feature enters: the dilation constant $c$ may be a global constant or merely constant on symplectic leaves.

We write $L$ for a symplectic leaf and $\omega_{L}$ for the symplectic form on the leaf.

Definition 2. Let $P$ be a Poisson manifold on which the leaves of maximal dimension are the level sets of a set of Casimir functions. The diffeomorphism $\varphi$ is called conformal (or $C$-conformal) if there exists a Casimir $C$ such that for all $f, g \in C^{\infty}(P)$,

$$
\{f \circ \varphi, g \circ \varphi\}=C\{f, g\} \circ \varphi .
$$

It is called special conformal if there exists a constant $c$ such that for all $f, g \in C^{\infty}(P)$,

$$
\{f \circ \varphi, g \circ \varphi\}=c\{f, g\} \circ \varphi .
$$

It is called Poisson if for all $f, g \in C^{\infty}(P)$,

$$
\{f \circ \varphi, g \circ \varphi\}=\{f, g\} \circ \varphi .
$$

Before describing the behavior of conformal diffeomorphisms, we obtain a useful characterization of the Poisson diffeomorphisms of a Poisson manifold $P$ by looking at their action on the symplectic leaves. Namely, they must map leaves to leaves symplectically. This behavior also characterizes the conformal Poisson diffeomorphisms. 
Proposition 9. Let $P$ be a Poisson manifold and $\varphi: P \rightarrow P$ be a diffeomorphism. Then $\varphi$ is Poisson if and only iffor all $z \in P, \varphi$ maps the symplectic leaf $L_{z}$ through $z$ diffeomorphically onto the leaf $L_{\varphi(z)}$ through $\varphi(z)$ and this map is symplectic, i.e.,

$$
\varphi^{*} \omega_{L_{\varphi(z)}}=\omega_{L_{z}}
$$

Proof. If $\varphi$ is a Poisson diffeomorphism, then the fact that it is Poisson guarantees that $\left.T_{z} \varphi\right|_{T_{z} L_{z}}: T_{z} L_{z} \rightarrow T_{\varphi(z)} L_{\varphi(z)}$ is surjective. Since $\varphi$ is a diffeomorphism, this map must also be injective. To see that $\varphi$ restricted to $L_{z}$ is surjective, note that every point $z^{\prime} \in L_{\varphi(z)}$ can be connected to $\varphi(z)$ by a sequence of Hamiltonian arcs. However, since, for every $h \in C^{\infty}(P), T \varphi \cdot X_{h \circ \varphi}=X_{h} \circ \varphi$, each Hamiltonian arc has a counterpart on $L_{z}$ that maps to it under $\varphi$. Thus, there exists a $z_{0}$ on $L_{z}$ such that $\varphi\left(z_{0}\right)=z^{\prime}$. We conclude $\varphi\left(L_{z}\right)=L_{\varphi(z)}$. Since $\varphi$ is a diffeomorphism, $\left.\varphi\right|_{L_{z}}$ must be one to one. Finally we show $\left.\varphi\right|_{L_{z}}$ is symplectic. Any tangent vector to $L_{z}$ can be written as $T \varphi^{-1} \cdot X_{h}(\varphi(z))$ for some function $h$ so that

$$
\begin{aligned}
\varphi^{*} & \omega_{\varphi(z)}\left(T \varphi^{-1} \cdot X_{h}(\varphi(z)), T \varphi^{-1} \cdot X_{g}(\varphi(z))\right) \\
& =\omega_{\varphi(z)}\left(X_{h}(\varphi(z)), X_{g}(\varphi(z))\right)=\{h, g\} \circ \varphi(z)=\{h \circ \varphi, g \circ \varphi\}(z) \\
& =\omega_{z}\left(X_{h \circ \varphi}(z), X_{g \circ \varphi}(z)\right)=\omega_{z}\left(T \varphi^{-1} \cdot X_{h}(\varphi(z)), T \varphi^{-1} \cdot X_{g}(\varphi(z))\right) .
\end{aligned}
$$

Conversely, suppose the leaves are symplectically diffeomorphic under $\varphi$. Fix $z \in P$ and $f, g \in C^{\infty}(P)$. To show $\{f \circ \varphi, g \circ \varphi\}(z)=\{f, g\} \circ \varphi(z)$ it suffices to show that $T_{z} \varphi$. $X_{g \circ \varphi}(z)=X_{g} \circ \varphi(z)$ since

$$
\mathbf{d}(f \circ \varphi)(z) \cdot X_{g \circ \varphi}(z)=\mathbf{d} f(\varphi(z)) \cdot T_{z} \varphi \cdot X_{g \circ \varphi}(z) .
$$

Now, for each $w_{\varphi(z)} \in T_{\varphi(z)} L_{\varphi(z)}$ there exists a unique $y_{z} \in T_{z} L_{z}$ with $T \varphi\left(y_{z}\right)=w$. Thus

$$
\begin{aligned}
\omega_{\varphi(z)}\left(T \varphi \cdot X_{g \circ \varphi}(z), w_{\varphi(z)}\right) & =\varphi^{*}\left(\omega_{\varphi(z)}\right)\left(X_{g \circ \varphi}(z), y_{z}\right)=\omega_{z}\left(X_{g \circ \varphi}(z), y_{z}\right) \\
& =\mathbf{d}(g \circ \varphi)(z) \cdot y_{z}=\mathbf{d} g(\varphi(z)) \cdot w_{\varphi(z)},
\end{aligned}
$$

as required.

Proposition 10. The diffeomorphism $\varphi$ is conformal iff it maps leaves to leaves of the same dimension and is conformal-symplectic on them; i.e., iff for each leaf L there is a constant c such that

$$
\varphi^{*} \omega_{\varphi(L)}=c \omega_{L}
$$

It is special conformal iff c can be chosen to be independent of L. It is Poisson iff $c \equiv 1$.

Proof. The proof is a straightforward modification of Proposition 9.

In the case that the leaves of $P$ are not defined by Casimirs, one can take Proposition 10 as the definition of a conformal Poisson diffeomorphism.

We also have the classes of vector fields whose flows are Poisson or (special) conformal; their defining equations are obtained by differentiating Eqs. (4.1) and (4.2). $X$ is a conformal, 
special conformal, or Poisson vector field, if for all $f, g \in C^{\infty}(P)$,

$$
X\{f, g\}=\{X f, g\}+\{f, X g\}-c\{f, g\},
$$

where $c$ is constant on leaves, constant, or zero.

One can also consider the leaf-preserving (conformal) Poisson diffeomorphisms and vector fields, giving six classes of diffeomorphisms.

Example 2 (Skew product Hamiltonian systems). Let $(M,-\mathrm{d} \theta)$ be an exact symplectic manifold, let $N$ be any manifold, and let $P=M \times N$ where the leaves are $M \times$ constant. Let $H: P \rightarrow \mathbb{R}$ be a Hamiltonian. We extend $X_{H}$ and $Z$ to $P$ by letting them be zero in the $N$ component. Let $Y$ be any vector field on $N$, which we extend to $P$ by letting it be zero in the $M$ component. Let $C: N \rightarrow \mathbb{R}$ be any function. Then the vector field

$$
X^{C}:=X_{H}+C(z) Z+Y
$$

is $C$-conformal. We shall see in Proposition 14 that this describes all conformal vector fields on $P$. In coordinates $(q, p, y)$ we have

$$
\dot{q}=H_{p}(q, p, y), \quad \dot{p}=-H_{q}(q, p, y)+C(y) p, \quad \dot{y}=Y(y) .
$$

By the Darboux-Weinstein theorem [22], all conformal Poisson systems take this form in any neighborhood in which the leaves have constant dimension. In the Poisson case, $C \equiv 0$, this kind of vector field is called a "skew product Hamiltonian system". We also note that time-dependent conformal Hamiltonian systems can be regarded as autonomous conformal Poisson systems in the extended phase space $M \times \mathbb{R}$.

Proposition 11. The conformal, special conformal, and Poisson diffeomorphisms each form a group, with their vector fields as Lie algebras. Each has the leaf-preserving diffeomorphisms as a subgroup. The Poisson vector fields form an ideal in the special conformal and in the conformal Lie algebras.

Proof. By iterating the definitions, it is easy to check that the conformal, special conformal and Poisson diffeomorphisms form a group. It is also obvious that the composition of two leaf preserving diffeomorphisms is again leaf preserving. Finally we check that the subalgebra of Poisson vector fields is an ideal inside the conformal and special conformal vector fields. Let $X$ be a Poisson vector field and $X^{C}$ be conformal with Casimir $C$. Then,

$$
\begin{aligned}
{\left[X^{C}, X\right]\{f, g\}=} & \left(X^{C} X-X X^{C}\right)\{f, g\} \\
= & X^{C}(\{X f, g\}+\{f, X g\})-X\left(\left\{X^{C} f, g\right\}+\left\{f, X^{C} g\right\}-C\{f, g\}\right) \\
= & \left\{X^{C} X f, g\right\}+\left\{X f, X^{C} g\right\}-C\{X f, g\}+\left\{X^{C} f, X g\right\}+\left\{X^{C} f, X g\right\} \\
& +\left\{f, X^{C} X g\right\}-C\{f, X g\}-\left(\left\{X X^{C} f, g\right\}+\left\{X^{C} f, X g\right\}+\left\{X f, X^{C} g\right\}\right. \\
& \left.+\left\{f, X X^{C} g\right\}-C\{X f, g\}-C\{f, X g\}\right) \\
= & \left\{\left[X^{C}, X\right] f, g\right\}+\left\{f,\left[X^{C}, X\right] g\right\},
\end{aligned}
$$

as required. The same argument proceeds with $C$ replaced by a constant $c$. 
Proposition 12. The Hamiltonian vector fields form an ideal in the Lie algebra of conformal vector fields.

Proof. For all $f \in C^{\infty}(P)$, we have

$$
\begin{aligned}
{\left[X_{H}, X^{C}\right] f } & =X_{H} X^{C} f-X^{C} X_{H} f=\left\{X^{C} f, H\right\}-X^{C}\{f, H\} \\
& =\left\{X^{C} f, H\right\}-\left(\left\{X^{C} f, H\right\}+\left\{f, X^{C} H\right\}-C\{f, H\}\right) \\
& =C\{f, H\}-\left\{f, X^{C} H\right\}=\left\{f, C H-X^{C} H\right\},
\end{aligned}
$$

and therefore

$$
\left[X^{C}, X_{H}\right]=X_{X^{C} H-C H} .
$$

Conformal vector fields have special properties with respect to the foliations of $P$ by the symplectic leaves. In particular, at regular points of the foliation, they drop to the leaf space. Since this will be developed further when we consider conformal vector fields with symmetry, it is useful to recall some general concepts from the theory of foliations (see [17 pp. 34-35]). Let $M$ be a smooth $n$-dimensional manifold on which we have a codimension $q$, smooth foliation $F$ with corresponding $(n-q)$-dimensional distribution $P$. Denote the vector fields tangent to the foliation, with values in $P$, by $\mathfrak{X}_{F}$.

Definition 3. A function $f$ on $M$ is basic if, for every $Y \in \mathfrak{X}_{F}, Y(f)=0$.

Definition 4. An open set $U$ in $M$ is simple if the foliation, restricted to $U$ is generated by the inverse images of a submersion $\pi: U \rightarrow \bar{U}$ where $\bar{U}$ is a quotient manifold of dimension $q$. On such a simple open set, there are local coordinates adapted to the foliation $\left(x_{1}, \ldots, x_{n-q}, y_{1}, \ldots, y_{q}\right)$ such that the leaves are obtained by setting the last $q$ coordinates to a constant.

Then it is clear that every basic function on a simple open set $U$ is projectable to a smooth function on $\bar{U}$. Finally we have

Definition 5. A vector field $X$ on $M$ is foliate if $[X, Y] \in \mathfrak{X}_{F}$ for all $Y \in \mathfrak{X}_{F}$.

Foliate vector fields have the property that on every simple open neighborhood, $U$, they drop to the quotient manifold $\bar{U}$. In terms of adapted local coordinates, the last $q$ components of a foliate vector field depend only on the variables $y_{1}, \ldots, y_{q}$.

Applying these definitions to the irregular foliation of $P$ by symplectic leaves we have

Proposition 13. $X^{C}$ is a foliate vector field.

Proof. $\left[X^{C}, X_{H}\right]$ is Hamiltonian from Eq. (4.6), and hence tangent to the symplectic leaves.

Therefore, in any simple open neighborhood, $X^{C}$ drops to the local space of leaves. In the case that the symplectic leaves of maximal dimension are the level sets of the Casimir 
functions $C_{1}, \ldots, C_{q}$, the union of these leaves is a simple open neighborhood and the Casimirs induce coordinates on the space of leaves. The dropped vector field can be written in coordinates as

$$
\dot{C}_{j}=f_{j}\left(C_{1}, \ldots, C_{q}\right) .
$$

We can describe the conformal vector fields concisely if the manifold supports just one special conformal vector field.

Proposition 14. Let $Z$ be a special conformal vector field with $c=1$. Then the Lie algebra of conformal vector fields is the set

$$
\{X+C Z \mid X \text { Poisson, C any Casimir }\} \text {. }
$$

Proof. Let $X^{C}$ be any $C$-conformal vector field. Then for all $f, g \in C^{\infty}(P)$,

$$
\begin{aligned}
& \left(X^{C}-C Z\right)\{f, g\}-\left\{\left(X^{C}-C Z\right) f, g\right\}-\left\{f,\left(X^{C}-C Z\right) g\right\} \\
& \quad=\left(X^{C}\{f, g\}-\left\{X^{C} f, g\right\}-\left\{f, X^{C} g\right\}\right)-C(Z\{f, g\}-\{Z f, g\}-\{f, Z g\}) \\
& \quad=(C-C)\{f, g\}=0
\end{aligned}
$$

(using the Leibniz rule and that $C$ is a Casimir), showing that $X:=X^{C}-C Z$ is Poisson.

On the other hand, if $Z$ is $C$-conformal but happens to be Poisson on some leaves (i.e., if $\left.C\right|_{L}=0$ for some leaves $L$ ), then $X+D Z, D$ any Casimir, is $D C$-conformal and hence also Poisson on these leaves. Such a $Z$ could not then be used to generate all conformal vector fields. We are not sure if manifolds can support conformal but not special conformal vector fields, however.

In many instances, the symplectic leaves are pairwise non-symplectomorphic, so Poisson vector fields must preserve the leaves; if, in addition, $H^{1}(L)=0$, then we can take $X=X_{H}$ in Eq. (4.7) for some Hamiltonian function $H$.

We do not have a characterization of those Poisson manifolds which support conformal vector fields. However, the following important example includes the canonical and Lie-Poisson cases, and has the feature that $Z$ is symmetric under the full general linear group.

Example 3 (Homogeneous Poisson brackets). Let $P=\mathbb{R}^{n}$ with a Poisson bracket homogeneous of degree $k$, i.e.,

$$
\left\{x_{i}, x_{j}\right\} \circ d=\alpha^{k}\left\{x_{i}, x_{j}\right\},
$$

where $d: x \mapsto \alpha x$ is a dilation. Then for $k \neq 2$,

$$
Z=\frac{1}{2-k} \sum_{i} x_{i} \frac{\partial}{\partial x_{i}}
$$

is a special conformal vector field with $c=1$. 
It is sufficient to check Eq. (4.5) for $f=x_{i}, g=x_{j}$. For $X=\sum x_{i}\left(\partial / \partial x_{i}\right)$ we have

$$
X\left\{x_{i}, x_{j}\right\}=\sum x_{k} \frac{\partial\left\{x_{i}, x_{j}\right\}}{\partial x_{k}}=\left.\frac{\partial}{\partial \alpha}\left(\left\{x_{i}, x_{j}\right\} \circ d\right)\right|_{\alpha=1}=\left.\frac{\partial}{\partial \alpha} \alpha^{k}\left\{x_{i}, x_{j}\right\}\right|_{\alpha=1}=k\left\{x_{i}, x_{j}\right\},
$$

and

$$
\left\{X x_{i}, x_{j}\right\}+\left\{x_{i}, X x_{j}\right\}-c\left\{x_{i}, x_{j}\right\}=(2-c)\left\{x_{i}, x_{j}\right\} .
$$

Therefore, $X$ is $(2-k)$-conformal, which gives the result.

Note that for $k=2$, this vector field is in fact Poisson. However, some quadratic Poisson brackets do have conformal vector fields.

Example 4. On $P=\left(\mathbb{R}^{+}\right)^{3},\left\{x_{i}, x_{j}\right\}=x_{i} x_{j}$, the vector field $\frac{1}{2} \sum x_{i} \log x_{i}\left(\partial / \partial x_{i}\right)$ is 1-conformal.

Another source of examples is provided by the reduction theorem in the next section.

\section{Symmetric conformal vector fields on Poisson manifolds}

In this section we consider the consequences of the conformal vector field on $P$ being symmetric with respect to a Poisson action of a Lie group $G$. First we show in Theorem 3 that the symmetric conformal vector field drops to a vector field on $P / G$ which is conformal with respect to the quotient Poisson structure. Second, in the case that the action admits an equivariant momentum map, the vector field also drops to another conformal vector field (Theorem 4), making the situation analogous to the symplectic case of a dual pair. This second quotient space is obtained by identifying points on the level sets of $\mathbf{J}$ on the same symplectic leaf. (This result seems to be new even in the non-conformal, i.e. Poisson, case.) Since each of these dropped vector fields is conformal they both drop to their respective leaf spaces as in Proposition 13.

Theorem 3 (Conformal Poisson reduction). Let $X^{C}$ be a C-conformal vector field on the Poisson manifold $P$ that is symmetric relative to a Lie group $G$ which acts on $P$ by Poisson diffeomorphisms. Assume that $G$ acts in such manner that the quotient space is a smooth manifold denoted as $P / G$. Then the reduced vector field on $P / G$ is conformal relative to the quotient Poisson structure.

Proof. Let $\pi: P \rightarrow P / G$ denote the quotient map. Let $X_{P / G}$ be the unique vector field that satisfies $T \pi \cdot X^{C}=X_{P / G} \circ \pi$. Let $f, g \in C^{\infty}(P / G)$ and $F=f \circ \pi, G=g \circ \pi$. Notice that $X_{P / G}(f)([z])=X^{c}(F)(z)$ so that

$$
\begin{aligned}
X_{P / G}\{f, g\}([z])= & X^{C}\{F, G\}(z)=\left\{X^{C}(F), G\right\}(z)+\left\{F, X^{C}(G)\right\}(z)-C\{F, G\}(z) \\
= & \left\{X_{P / G}(f) \circ \pi, g \circ \pi\right\}(z)+\left\{f \circ \pi, X_{P / G}(g) \circ \pi\right\}(z) \\
& -C\{f \circ \pi, g \circ \pi\}(z) \\
= & \left\{X_{P / G}(f), g\right\}([z])+\left\{f, X_{P / G}(g)\right\}([z])-C([z])\{f, g\}([z]) .
\end{aligned}
$$


In the last line we have used the fact that the Casimir $C$ must be $G$-invariant and hence defines a function $C([z])$. This follows since the left-hand side of the second equality is $G$-invariant and so are the first and second terms of the right-hand side, thus implying that $C\{F, G\}$ is a $G$-invariant function. Since $\{F, G\}$ is $G$-invariant, we conclude that $C$ is $G$-invariant as well. (This would not be true for all Casimirs $C$ unless the action were Hamiltonian.) The last equality shows that $X_{P / G}$ is $C([z])$-conformal relative to the reduced Poisson bracket.

Note that by taking $C=$ constant, the same result holds for special conformal vector fields with symmetry.

An example of reduction of a symmetric conformal vector field is given by reducing the Liouville vector field $Z$ on $T^{*} Q$ by a cotangent lifted symmetry, producing a conformal vector field on $T^{*} Q / G$. We can obtain a simple decomposition of the reduced vector field if we split the vector bundle $T^{*} Q / G$ into a Whitney sum (a direct sum of two vector bundles with the same base).

Example 5 (Reduction of the Liouville vector field). Let $\mathfrak{A}$ be a connection on the principal bundle $\pi_{Q}: Q \rightarrow Q / G$, and let $\pi: T^{*} Q \rightarrow T^{*} Q / G$ as in Theorem 3. We make use of the following bundle isomorphism [13]:

$$
\frac{T^{*} Q}{G} \simeq T^{*}\left(\frac{Q}{G}\right) \oplus \tilde{\mathfrak{g}}^{*}
$$

which is dual to the vector bundle isomorphism of the reduced tangent bundle, $T Q / G \simeq$ $T(Q / G) \oplus \tilde{\mathfrak{g}}$ given by $\varphi_{\mathfrak{A}}:\left[v_{q}\right] \mapsto T_{q} \pi_{Q} \cdot v_{q} \oplus\left[q, \mathfrak{A}(q)\left(v_{q}\right)\right]$. Then

$$
\varphi_{\mathfrak{A}}^{-1}\left(v_{[q]} \oplus[q, \xi]\right)=\left[\operatorname{hor}_{q} v_{[q]}+\xi_{Q}(q)\right],
$$

where [, ] denotes an equivalence class for the group action on the appropriate space. The desired isomorphism, Eq. (5.1), is then given by

$$
\left(\varphi_{\mathfrak{A}}^{-1}\right)^{*}\left(\left[\alpha_{q}\right]\right)=\operatorname{hor}_{q}^{*} \alpha_{q} \oplus\left[q, \mathbf{J}\left(\alpha_{q}\right)\right]
$$

We compute the flow of $T\left(\left(\varphi_{\mathfrak{A}}^{-1}\right)^{*}\right) \circ T \pi \cdot Z$, which is a well-defined vector field on the Whitney sum bundle (Eq. (5.1)) since $Z$ is $G$-invariant. The flow of $Z$ on $T^{*} Q$ is $\psi_{t}\left(\alpha_{q}\right)=$ $\mathrm{e}^{c t} \alpha_{q}$ so that

$$
\left(\varphi_{\mathfrak{A}}^{-1}\right)^{*} \circ \pi\left(\psi_{t}\left(\alpha_{q}\right)\right)=\operatorname{hor}_{q}^{*}\left(\mathrm{e}^{c t} \alpha_{q}\right) \oplus\left[q, \mathbf{J}\left(\mathrm{e}^{c t} \alpha_{q}\right)\right]=\mathrm{e}^{c t} \operatorname{hor}_{q}^{*}\left(\alpha_{q}\right) \oplus \mathrm{e}^{c t}\left[q, \mathbf{J}\left(\alpha_{q}\right)\right] .
$$

Observe that this is the flow of the vector field which is the Liouville vector field on $T^{*}(Q / G)$ in the first factor, and the radial vector field on each fiber of $\tilde{\mathfrak{g}}^{*}$ in the second factor. The vector field projects to 0 on the base manifold $Q / G$.

A conformal vector field with a symmetry admitting a momentum map on a Poisson manifold satisfies an analog of the momentum equation (2.10) for exact symplectic manifolds. This is made precise in the next proposition. Since it makes no use of Hamiltonian 
functions, it also extends Proposition 7 to the case $H^{1}(M) \neq 0$, albeit with a slightly weaker conclusion.

Proposition 15 (Conformal Poisson momentum equation). Suppose we have a left action of $G$ on $P$ for which there exists an equivariant momentum map $\mathbf{J}: P \rightarrow \mathfrak{g}^{*}$. Let $X^{C}$ be a $G$-invariant conformal vector field. Then the following equation is satisfied for all $\xi \in \mathfrak{g}$, and for all $h \in C^{\infty}(P)$,

$$
\left\{X^{C}(J(\xi))-C J(\xi), h\right\}=0,
$$

where as usual $J(\xi)=\langle\mathbf{J}, \xi\rangle$. In other words there is a Casimir $X^{C}(J(\xi))-C J(\xi)$ corresponding to each Lie algebra element $\xi$. It follows that there exists a linear map $\psi: \mathfrak{g} \rightarrow \operatorname{Cas}(P)$ defined by

$$
\psi(\xi):=X^{C}(J(\xi))-C J(\xi)
$$

and its companion $\boldsymbol{\psi}: P \rightarrow \mathfrak{g}^{*}$ defined by

$$
\langle\psi(z), \xi\rangle=\psi(\xi)(z)
$$

Proof. We have

$$
\begin{aligned}
\left\{X^{C}(J(\xi)), h\right\} & =X^{C}\{J(\xi), h\}-\left\{J(\xi), X^{C}(h)\right\}+C\{J(\xi), h\} \\
& =-X^{C}\left(\xi_{P}(h)\right)+\xi_{P}\left(X^{C}(h)\right)+C\{J(\xi), h\} \\
& =\left[\xi_{P}, X^{C}\right](h)+C\{J(\xi), h\}=C\{J(\xi), h\},
\end{aligned}
$$

from which the statement follows. Notice that we use the $G$-invariance of $X^{C}$ in the last equality. The existence and linearity of $\psi$ are now immediate.

Theorem 4. Let $X^{C}, G$, and $P$ be as in the preceding proposition. Let $F$ be the intersection of the foliations of $P$ by symplectic leaves and by level sets of the momentum $\mathbf{J}$. Then (i) $P / F$ is a Poisson manifold with respect to the quotient Poisson structure; (ii) $X^{C}$ drops to this quotient, and (iii) the dropped vector field is conformal with respect to the quotient Poisson structure.

Proof. Let $\pi: P \rightarrow P / F$ denote the quotient map, let $f_{1}, f_{2} \in C^{\infty}(P / F)$ and let $F_{1}=f_{1} \circ \pi, F_{2}=f_{2} \circ \pi$. We first show that the quotient Poisson bracket

$$
\left\{f_{1}, f_{2}\right\}_{P / F}:=\left\{F_{1}, F_{2}\right\}
$$

is well-defined, i.e. the right-hand side is constant on $F$. The tangent space to the foliation at any point is spanned by the Hamiltonian vector fields of the $G$-invariant functions since these are the only tangent vectors to the symplectic leaves that are also contained in the levels of $\mathbf{J}$. It therefore suffices to show that $X_{H}\left\{F_{1}, F_{2}\right\}=0$ for all such functions $H$, which will follow from $\left\{H,\left\{F_{1}, F_{2}\right\}\right\}=0$. But

$$
\left\{H,\left\{F_{1}, F_{2}\right\}\right\}=\left\{\left\{H, F_{1}\right\}, F_{2}\right\}+\left\{F_{1},\left\{H, F_{2}\right\}\right\} .
$$


Since $X_{H}$ is tangent to both the symplectic leaves and the level surfaces of $\mathbf{J}$, and $F_{i}$ is constant on this intersection, we must have

$$
\mathbf{d} F_{i} \cdot X_{H}=0,
$$

which implies $\left\{H, F_{i}\right\}=0$ and hence $X_{H}\left\{F_{1}, F_{2}\right\}=0$. Since the quotient bracket is well-defined it is necessarily a Poisson bracket. To establish (ii), we have to show that $X^{C}$ is foliate, which is equivalent to $\mathbf{L}_{X_{H}} X^{C}$ being tangent to the foliation for all $G$-invariant $H$. From Eq. (4.6), we have

$$
\mathbf{L}_{X_{H}} X^{C}=\left[X_{H}, X^{C}\right]=X_{C H-X^{C}} \cdot
$$

The two functions $X^{C} H$ and $C H$ are both $G$-invariant, so the right-hand side is tangent to the foliation. The calculation for (iii) is as for the previous proposition.

We can put local coordinates on $P / F$ by choosing a basis of Casimirs, $C_{1}, \ldots, C_{q}$ so that in a neighborhood $U$ of a regular point of $P$, the symplectic leaves are the level sets of the function $K=C_{1} \times \cdots \times C_{q}, K: U \rightarrow \mathbb{R}^{q}$. Then on $\pi(U)$ we have coordinates induced by the basic (Definition 3) functions on $P,(K, C)=\left(J\left(\xi_{1}\right), \ldots J\left(\xi_{k}\right), C_{1}, \ldots, C_{n}\right)$. In these coordinates the dropped vector field $X_{P / F}$ has the form:

$$
\dot{K}=f(K), \quad \dot{J}=C J+\psi
$$

for some vector field $f$ on $\mathbb{R}^{q}$. Recall that the momentum, $\mathbf{J}$ is only defined up to a Casimir. Therefore, if $C(z) \neq 0$ for all $z \in P$, then defining $\tilde{J}=J+\psi / C$ we have

$$
\dot{\tilde{J}}=C \tilde{J}
$$

i.e., the momentum equation is again linear and radial.

We consider two special cases. First, the case when $P$ is symplectic. The symmetric conformal vector field $X^{c}$ is then foliate with respect to the level sets of $\mathbf{J}$ and defines a vector field on $\mathfrak{g}^{*}$ given by

$$
X(\mu)=T \mathbf{J} \cdot X^{c}(z)
$$

for $z \in \mathbf{J}^{-1}(\mu)$. To see this, note that $X^{c}$ is foliate with respect to $\mathbf{J}$ if and only if for all $\mu \in \mathbf{J}(P) \subset \mathfrak{g}^{*}$, and for all $z_{1}, z_{2} \in \mathbf{J}^{-1}(\mu)$, we have

$$
T_{z_{1}} \mathbf{J} \cdot X^{c}\left(z_{1}\right)=T_{z_{2}} \mathbf{J} \cdot X^{c}\left(z_{2}\right) .
$$

However,

$$
T_{z_{1}} \mathbf{J} \cdot X^{c}\left(z_{1}\right)-T_{z_{2}} \mathbf{J} \cdot X^{c}\left(z_{2}\right) c \mathbf{J}\left(z_{1}\right)+\psi\left(z_{1}\right)-\left(c \mathbf{J}\left(z_{2}\right)+\psi\left(z_{2}\right)\right)=\psi\left(z_{1}\right)-\psi\left(z_{2}\right)=0,
$$

where the last equality follows from the fact that each Casimir $\psi(\xi)$ is constant on $P$. Thus for symplectic manifolds, every symmetric conformal vector field is bifoliate on $P$ and therefore defines a dropped vector field on each leg of the dual pair $P / G \leftarrow P \rightarrow \mathfrak{g}^{*}$. The stronger hypothesis of Proposition 7, that $X^{c}$ be conformal Hamiltonian, is not required. 
The Casimir $\psi$ in Eq. (5.5) is a constant, but as in Eq. (5.6) this constant can be eliminated when $c \neq 0$ by a shift in $J$. On the other hand, if $X^{c}=X_{H}^{c}$, then dropped vector field is always linear and radial, from Proposition 7.

Second, if $P$ is Poisson and the levels of $\mathbf{J}$ are contained in the symplectic leaves, then the foliation $F$ of $P$ reduces to the foliation $\mathbf{J}$ so Theorem 4 tells us that $T \mathbf{J} \cdot X^{C}$ defines a vector field on $\mathfrak{g}^{*}$. There is a sufficient condition for this to hold, namely that the image of the $G$-invariant functions under the Poisson tensor has full rank.

There are many examples of conformal Poisson systems with symmetry. Often the radial vector field $R^{c}$ is conformal, and is invariant under any linear group action, as in the following example.

Example 6 (Lie-Poisson). Consider the radial vector field on $\mathfrak{g}^{*}$ given by $R^{c}(\mu)=c \mu$. Denote the natural coadjoint action $G \times \mathfrak{g}^{*} \rightarrow \mathfrak{g}^{*}$ by

$$
g \cdot \mu:=\operatorname{Ad}_{g^{-1}}^{*} \mu .
$$

This action is Hamiltonian with momentum map given by $\mathbf{J}(\mu)=-\mu . R^{c}$ is $G$-invariant since

$$
\varphi_{g}^{*} R^{c}(\mu)=T \varphi_{g^{-1}} \cdot R^{c}(g \cdot \mu)=\left.\frac{\mathrm{d}}{\mathrm{d} t}\right|_{t=0} g^{-1} \cdot(g \cdot \mu+\operatorname{tcg} \cdot \mu)=c \mu=R^{c}(\mu) .
$$

We then have $T \mathbf{J} \cdot R^{c}=-R^{c}=c \mathbf{J}$ as required.

Further examples of Poisson manifolds supporting symmetric conformal vector fields are produced by symplectic reduction by stages $[11,12]$. To do this we first reduce Poisson and then attempt to induce a momentum map for the second stage action on a reduced Poisson manifold. Since $[11,12]$ only deal with symplectic reduction we first develop the necessary theory.

Let $M$ be a group which acts on the Poisson manifold $P$ by Poisson maps admitting a momentum map $\mathbf{J}: P \rightarrow \mathfrak{m}^{*}$. Suppose $N$ is a normal subgroup of $M$, which therefore also has an action admitting the momentum map $\mathbf{J}_{N}=\iota_{\mathfrak{n}}^{*} \mathbf{J}$. It is easy to see that $P / N$ is a Poisson manifold and $M / N$ acts by Poisson morphisms. Under what conditions does this action admit a momentum map? The quotient action is defined by the equation,

$$
[m] \cdot[z]=[m \cdot z]
$$

where the $[m]$ is the equivalence class of the point $m \in M$ in $M / N$. Alternatively we write $[m]=\pi^{M}(m)$, where $\pi^{M}: M \rightarrow M / N$. Note that this is the quotient map for the left action of $N$ on $M$. Take a point in the Lie algebra of the quotient group, $[\xi] \in \mathfrak{m} / \mathfrak{n}$. Denoting by $\pi: P \rightarrow P / N$ the quotient map for the left action of $N$ on $P$, we have

Theorem 5. Let $P$ be a Poisson manifold on which the group $M$ acts by Poisson diffeomorphisms admitting an equivariant momentum map, $\mathbf{J}_{M}: P \rightarrow \mathfrak{m}^{*}$. Suppose $N$ is contained in the center of $M$. Then $P / N$ is a Poisson manifold on which $M / N$ acts admitting a momentum map $\mathbf{J}_{M / N}: P / N \rightarrow \mathfrak{m}^{*} / \mathfrak{n}^{*}$. 
Proof. The quotient action admits a momentum map if and only if for every $\xi \in \mathfrak{m}$, and every function $f \in C^{\infty}(P / N)$ we have

$$
[\xi]_{P / N}(f)=\{f, J([\xi])\}_{P / N}
$$

for some linear function $J: \mathfrak{m} / \mathfrak{n} \rightarrow C^{\infty}(P / N)$. Now, by definition of the quotient action, we have

$$
\begin{aligned}
{[\xi]_{P / N}(f) } & =\left.\frac{\mathrm{d}}{\mathrm{d} t}\right|_{t=0} f\left(\exp _{M / N} t[\xi] \cdot[z]\right)=\left.\frac{\mathrm{d}}{\mathrm{d} t}\right|_{t=0} f\left(\left[\exp _{M} t \xi\right] \cdot[z]\right) \\
& =\left.\frac{\mathrm{d}}{\mathrm{d} t}\right|_{t=0} F\left(\exp _{M} t \xi \cdot z\right)=\mathbf{d} F(z) \cdot \xi_{M}(z)=\left\{F,\left\langle\mathbf{J}_{M}, \xi\right\rangle\right\}(z) \\
& =\left\{f \circ \pi_{N}, J_{M}(\xi)\right\}(z),
\end{aligned}
$$

where $F=f \circ \pi_{N}$ and we have used the fact that $\pi^{M}$ is a group homomorphism and therefore

$$
\exp _{M / N} \circ T_{e} \pi^{M}=\pi^{M} \circ \exp _{M} .
$$

Comparing with Eq. (5.9), we see that for the $M / N$ action to admit a momentum map, we require $J_{M}(\xi)$ to be $N$-invariant for each $\xi \in \mathfrak{m}$. Infinitesimally, this requires that for all $\eta \in \mathfrak{n}$,

$$
\eta_{P}\left(J_{M}(\xi)\right)=0=\left\{J_{M}(\xi), J_{M}(\eta)\right\} .
$$

Now, from the assumption that the momentum map is $\mathrm{Ad}^{*}$-equivariant, it follows that

$$
\left\{J_{M}(\xi), J_{M}(\eta)\right\}=J([\xi, \eta]) .
$$

We can immediately conclude that if $[\xi, \eta]=0$ for all $\xi \in \mathfrak{m}$ and all $\eta \in \mathfrak{n}$ then the right-hand side of the above equation is zero since $J$ is a linear map. The statement of the theorem is now immediate.

Remark. Suppose $P=T^{*} Q$ with canonical symplectic form. The existence of an induced momentum map on the quotient Poisson manifold then requires that $\mathfrak{n}$ be contained in the center of $\mathfrak{m}$. This is because $J(\xi)\left(\alpha_{q}\right)=\left\langle\alpha_{q}, \xi_{Q}\right\rangle$ and so $J([\xi, \eta])=0$ if and only if $[\xi, \eta]_{Q}=0$. However, since the action of $M$ on $Q$ is presumed to be free, an infinitesimal generator vanishes if and only if the Lie algebra element is zero. Thus we require $[\xi, \eta]=0$ for all $\xi \in \mathfrak{m}$ and for all $\eta \in \mathfrak{n}$.

Example 7 (Reduction by stages). Starting with a cotangent bundle, we can produce a category of examples of conformal vector fields with symmetry on non-symplectic Poisson spaces which satisfy the hypotheses of Proposition 15. The idea is to half reduce the cotangent bundle by reducing by a central subgroup; the quotient will then act on the reduced space to produce the example. We start with $M \times T^{*} Q \rightarrow T^{*} Q$ acting by cotangent lift. Let $N$ be contained in the center. Let $H$ be an $M$-invariant function on $T^{*} Q$ so that $X_{H}^{c}$ is an $H$-invariant conformal vector field on $T^{*} Q$. Quotienting by the $N$ action we 
obtain by Theorem 3 a conformal vector field on the Poisson manifold $T^{*} Q / N$. In fact this manifold is diffeomorphic to the Whitney sum bundle $T^{*}(Q / N) \oplus \tilde{\mathfrak{n}}^{*}$ and carries a non-canonical bracket. Furthermore, the quotient group $M / N$ acts on it by Poisson maps and admits a momentum map $\mathbf{J}_{M / N}$ by Theorem 5 . It is straightforward to show that this action leaves the vector field $T \pi_{N} \cdot X_{H}^{c}$ invariant and so we have satisfied the hypotheses of Proposition 15.

Example 8 (The dual of a Lie algebroid). The conformal vector field on $T^{*} Q / G$ obtained in Example 5 by reduction is in fact a special case of a conformal vector field on the dual of a Lie algebroid. Recall that a Lie algebroid, $\mathcal{A}$, is a vector bundle over a manifold $M$ whose sections form a Lie algebra which satisfy, for any smooth function $f$ on $M$,

$$
[f \xi, \eta]=f[\xi, \eta]-(\rho(\eta) f) \xi
$$

for a bundle map called the anchor, $\rho: \mathcal{A} \rightarrow T M$, that maps sections of $\mathcal{A}$ homomorphically into vector fields on $M$. The dual of the algebroid, $\mathcal{A}^{*}$, then carries a Poisson structure which is uniquely determined by specifying the bracket on functions which are affine on the fibers of $\mathcal{A}^{*}$. If we let $f, g \in C^{\infty}(M), \xi, \eta \in \Gamma(\mathcal{A})$, then the bracket on $\mathcal{A}^{*}$ is determined by

$$
\{f, g\}=0, \quad\{f, \xi\}=\rho(\xi)(f), \quad\{\xi, \eta\}=[\xi, \eta]
$$

where $\xi$ and $\eta$ are regarded as linear functions on the fibers of $\mathcal{A}$ via the natural pairing. Now consider the vector field $R^{c}$ tangent to the fibers of $\mathcal{A}^{*}$ and uniquely determined by its action on the functions linear on the fibers giving $R^{c}(\xi)=c \xi$. In coordinates $(q, \lambda)$ on $\mathcal{A}$, and inducing coordinates $(q, \mu)$ on $\mathcal{A}^{*}$, we have

$$
R^{c}(q, \mu)=c \mu \frac{\partial}{\partial \mu} .
$$

It suffices to check the conformal identity, Eq. (4.5) for pairs of functions, $\{f, g\},\{f, \xi\}$, and $\{\xi, \eta\}$ in the defining relations of the bracket since both the bracket and the vector field are determined by their action on affine functions. First, since $f$ and $g$ are constant on the fibers of $\mathcal{A}^{*}$, we have $R^{c}(f)=0$ so $R^{c}\{f, g\}=0=\left\{R^{c}(f), g\right\}+\left\{f, R^{c}(g)\right\}-c\{f, g\}$ since each term is zero. Furthermore, notice that

$$
R^{c}(\rho(\xi)(f))=0
$$

since $\rho(\xi)(f)$ is also constant on the fibers. Therefore,

$$
R^{c}\{f, \xi\}=0=\left\{R^{c} f, \xi\right\}+\left\{f, R^{c} \xi\right\}-c\{f, \xi\}=0+\{f, c \xi\}-c\{f, \xi\}=0 .
$$

Finally,

$$
R^{c}\{\xi, \eta\}=R^{c}[\xi, \eta]=c[\xi, \eta]=\left\{R^{c} \xi, \eta\right\}+\left\{\xi, R^{c} \eta\right\}-c\{\xi, \eta\}
$$

as required.

In the case that $\mathcal{A}=T^{*} Q / G$, the vector field $R^{c}$ is in fact the projection of $c Z$, the Liouville vector field, to the quotient by the $G$ action. 


\section{Open problems}

We close with some remarks and open problems.

1. In Example 5 we computed the reduction of the Liouville vector field $Z$ to $T^{*} Q / G$. What is the analogue of the reconstruction Theorem 2 in this case?

2. Symplectic manifolds have only one leaf and hence the conformal and special conformal vector fields coincide on them. Reducing therefore only leads to another special conformal vector field, even though the Poisson manifold it lives on may also support non-special ones. Reducing simple mechanical systems with symmetry therefore leads to a range of natural special conformal systems on various Poisson manifolds. If one takes the point of view that non-canonical Poisson manifolds arise primarily by reduction, then one must ask: Does non-special conformal dynamics ever arise naturally in mechanics?

3. One can ask if it is possible for a system to do anything else to a symplectic structure, for example dissipate it at a non-constant rate. This is not possible when $\operatorname{dim} M>2$, for it requires $\omega(u, v)$ and $\left(\mathbf{L}_{X} \omega\right)(u, v)$ to have different signs for all vector fields $u, v$, which implies $\mathbf{L}_{X} \omega=f \omega$ for some function $f: M \rightarrow \mathbb{R}$ with $f(x) \leq 0$ for all $x \in M$. But then $0=\mathrm{d}(f \omega)=\mathrm{d} f \wedge \omega \Rightarrow f=$ constant [9], and we are back in the conformal case. There remains a possibility for the symplectic structure to interact with some other structure on the manifold.

4. Given a vector field on the space of symplectic leaves of a Poisson manifold, when does it lift to a (conformal) Poisson vector field?

5. Which Poisson manifolds support conformal vector fields? Can they support conformal but not special conformal vector fields? Does the description of conformal vector fields in Proposition 14 extend to conformal Poisson maps?

6. What are the characteristic features of conformal dynamics, i.e. what are its homomorphism invariants?

\section{Acknowledgements}

We are grateful to the Marsden Fund of the Royal Society of New Zealand for financial support.

\section{References}

[1] H. Anzai, Ergodic skew product transformations on the torus, Osaka Math. J. 3 (1951) 83-99.

[2] A. Banyaga, The structure of classical diffeomorphism groups, Mathematics and Its Applications, Vol. 400, Kluwer Academic Publishers, Dordrecht, 1997.

[3] A.M. Bloch, P.S. Krishnaprasad, J.E. Marsden, T.S. Ratiu, The Euler-Poincaré equations and double bracket dissipation, Commun. Math. Phys. 175 (1996) 1-42.

[4] K. Burns, A. Wilkinson, Stable ergodicity of skew products, Ann. Sci. Ecole Norm. Sup. 32 (1999) (6) (4) 859-889.

[5] E. Cartan, Les groupes de transformation continus, infinis, simples, Ann. Sci. Ecole Norm. Sup. 26 (1909) 93-161. 
[6] U. Dessler, Symmetry property of the Lyapunov spectra of a class of dissipative dynamical systems with viscous damping, Phys. Rev. A 38 (1988) 2103.

[7] V. Guillemin, D. Quillen, S. Sternberg, The classification of the complex primitive infinite pseudogroups, Proc. Nat. Acad. Sci. 55 (1966) 687-690.

[8] M. Jonsson, Dynamics of polynomial skew products on $\mathbb{C}^{2}$, Math. Ann. 314 (3) (1999) $403-447$.

[9] S. Kobayashi, Transformation Groups in Differential Geometry, Springer, Berlin, 1972.

[10] P. Libermann, C.-M. Marle, Symplectic Geometry and Analytical Mechanics, Reidel, Dordrecht, 1987.

[11] J.E. Marsden, G. Misiołek, M. Perlmutter, T. Ratiu, Symplectic reduction for semidirect products and central extensions, Diff. Geom. Appl. 9 (1998) 173-212.

[12] J.E. Marsden, G. Misiołek, M. Perlmutter, T. Ratiu, Symplectic reduction by stages, Preprint.

[13] J.E. Marsden, M. Perlmutter, The orbit bundle picture of cotangent bundle reduction, CR Math. Rep. Acad. Sci., Canada 22 (2000) 33-54.

[14] J.E. Marsden, T.S. Ratiu, Introduction to Mechanics and Symmetry, 2nd Edition, Springer, New York, 1999.

[15] R.I. McLachlan, G.R.W. Quispel, What kinds of dynamics are there? Geometric integration and Lie pseudogroups, in preparation.

[16] R.I. McLachlan, G.R.W. Quispel, N. Robidoux, Unified approach to Hamiltonian systems, Poisson systems, gradient systems, and systems with Lyapunov functions or first integrals, Phys. Rev. Lett. 81 (1998) 2399-2403.

[17] P. Molino, Riemannian Foliations, Birkhäuser, Boston, 1988.

[18] J. Moser, On quadratic symplectic mappings, Math. Z. 216 (1994) 417-430.

[19] S. Shnider, Classification of the real infinite simple and real infinite primitive Lie algebras, J. Diff. Geom. 4 (1970) 81-89.

[20] I.M. Singer, S. Sternberg, On the infinite groups of Lie and Cartan. I, J. Anal. Math. 15 (1965) 1-114.

[21] O. Stormark, Lie's Structural Approach to PDE Systems, Cambridge University Press, Cambridge, 2000.

[22] A. Weinstein, The local structure of Poisson manifolds, J. Diff. Geom. 18 (1983) 523-557.

[23] M.P. Wojtkowski, C. Liverani, Conformally symplectic dynamics and symmetry of the Lyapunov spectrum, Commun. Math. Phys. 194 (1998) 47-60.

[24] E. Cartan, Euvres Complète d'Elie Cartan, Springer, Berlin, 1984. 